\title{
ON THE PELVIC EQUIVALENT OF THE SENSORY VAGUS. FURTHER CONTRIBUTIONS TO THE STUDY OF THE SACRO-BULBAR CONNECTIONS AND THEIR RELA- TION TO THE BULBAR VASOMOTOR CENTERS
}

\author{
MASARU KURU ${ }^{1}$ \\ Neurological Laboratory of M. Kuru's Surgical Clinic, \\ Kanazawa University School of Medicine
}

The vagus nerve contains besides somato- and viscero-motor fibers, somatoand viscero-sensory fibers. The nerve cells of the latter are situated, as is known, in the jugular and nodose ganglia, and their central fibers participating in the formation of the solitary fascicle descend towards the inferior part of the medulla. The solitary fascicle, seen in sharp contrast to the rather poorly medullated background in Pal-Weigert preparations, can be distinguished in all vertebrates. The upper-most part of this fascicle contains part of the taste mediating fibers from the glossopharyngeal, facial (intermedius) and trigeminal nerves and the lower half contains the centripetal half of the respiratory reflex arc.

The peripheral fibers of the sensory vagus innervate the external auditory canal, heart, lungs, oesophagus and stomach, but the destination of their caudal part is obscure and there are a number of viscera whose sensory innervation has decidedly no relation to the vagus. These are the distal part of the digestive tract and that part of the urogenital system that lies in the pelvic cavity which is phylogenetically a derivative of the former. The rôle of the hypogastric nerve in the conduction of visceral sensations from the pelvic organs is negligible (Fröhlich and Meyer (3)), and the nerve cells, whose peripheral branches via the sacral and pudendal plexuses innervate these pelvic organs, exist in the posterior root ganglia, and send their axonic processes (central branches) to the cord through the posterior roots of the 2nd, 3rd and 4th sacral segments. As to the intraspinal course and the termination of these fibers the anatomical findings were lacking, although a symptomatic analysis of chordotomy cases, suggests certain physiological information regarding them. Fibers mediating the sexual sensation together with those for pain ascend undoubtedly in the lateral funiculus, as bilateral chordotomy for relief of pain abolishes without fail libido and sexual orgasm (Foerster and Gagel (2)), while the sensations of bladder and rectal fullness seem to be transmitted by the

\footnotetext{
1 久留 勝

Received for publication November 29, 1950.
} 
posterior funiculus, since it is usually preserved even after a complete bilateral chordotomy (White (20)).

In tracing the ascending degenerations in Marchi-perparations of chordotomy cases, I (8) succeeded in 1940 to trace marked connections (tractus sacrobulbares) between the sacral cord and the medulla oblongata. These connections originate in the intermediate zone of the sacral and coccygeal cord (Kuru and Takase (10)), decussate in the anterior grey commissure (Nakamura (15)), ascend in the contralateral anterolateral fascicle and terminate in the juxtasolitary nucleus and other small grey matters dorsal and ventral to the spinal root of the trigeminus in the medulla. Meanwhile some of these enter Goll's

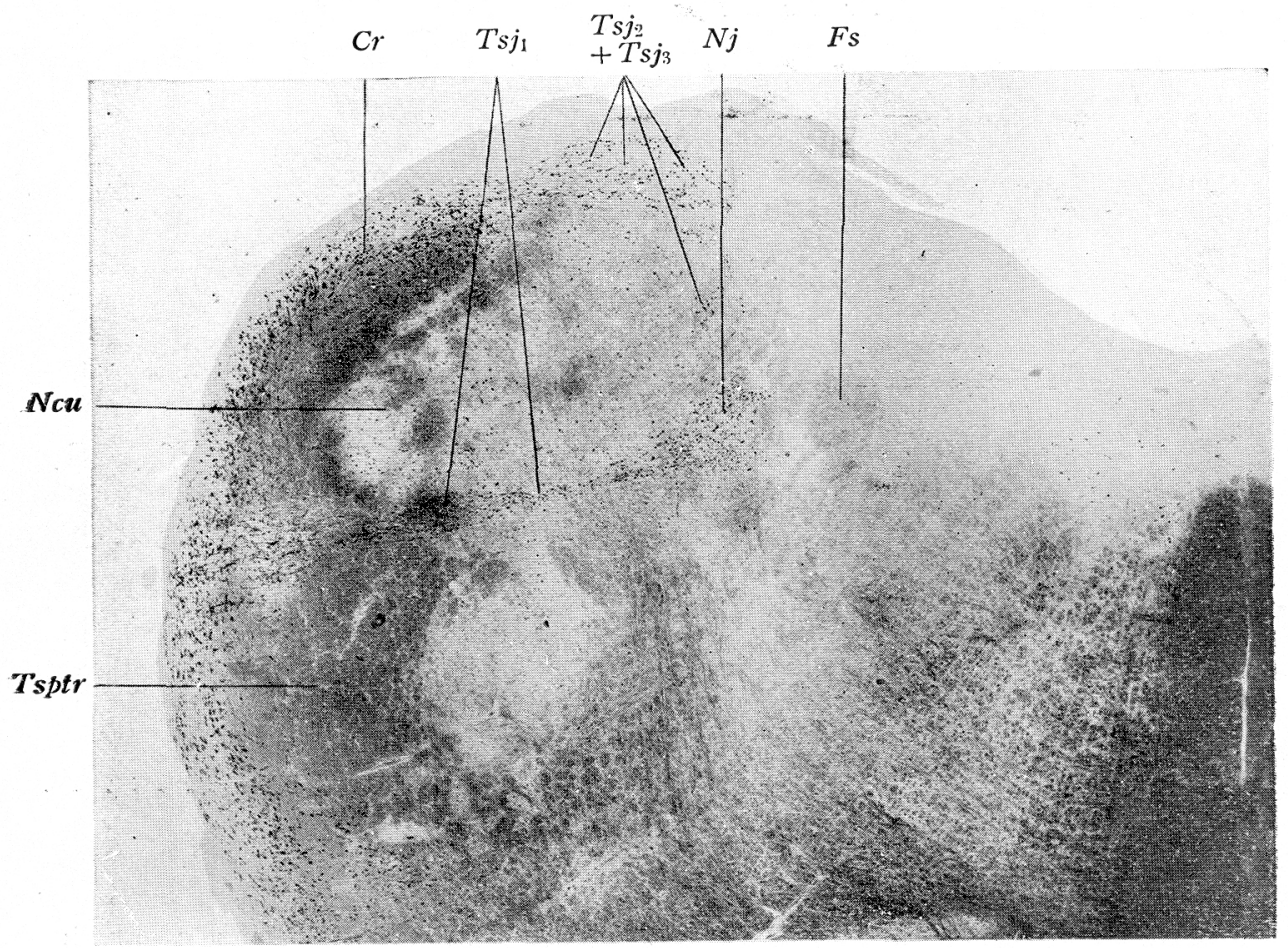

Fig. 1. Transverse section of the medulla at the level of the middle of the inferior olive. Case of anterolateral chordotomy (posterior funiculus intact). A conspicuous mass of degenerated fibers (Tractus spino-juxtasolitarialis- $T s j_{1}$ ) bends rectangularly between the cuneate nucleus $(N c u)$ and the spinal tract of the trigeminus ( Tsptr) and terminates in the nucleus (Nucleus juxtasolitarius $-N j$ ) just lateral to the solitary fascicle ( $F s$ ). Dorsal to this, another group of degenerated fibers is visible $\left(T s j_{2}+T s j_{3}\right)$, a part of which has ascended penetrating Goll's nucleus, while the other has emerged from the corpus restiforme $(\mathrm{Cr})$. Marchi-stain, $50 \mu$ thick.

nucleus (fibrae transclavares), gather at its medio-dorsal part and ascend further to its rostral continuation, which, lying near the lateral margin of the dorsal nucleus of the vagus, is directly dorsal to the rostral half of the nucleus juxtasolitarius (fig. 1). At that time, however, it could not be ascertained whether 
this part should be included in the juxtasolitary nucleus or in the rostral projection of the proper gracile nucleus, for in tracing the preparations in lesions of the lumbo-sacral posterior roots (cases of Dogliotti's subarachnoidal alcohol injection), I found the terminations of considerable numbers of fine-myelinated degenerated fibers in this rostral continuation of Goll's nucleus (fig. 2). This part, extending up to the middle part of the medulla oblongata, has been regarded by some authors (Marburg (14), Goldowski (5)) as the most caudal part of the triangular nucleus of the vestibular nerve, while Ziehen (22) insisted on its belonging to the gracile nucleus.

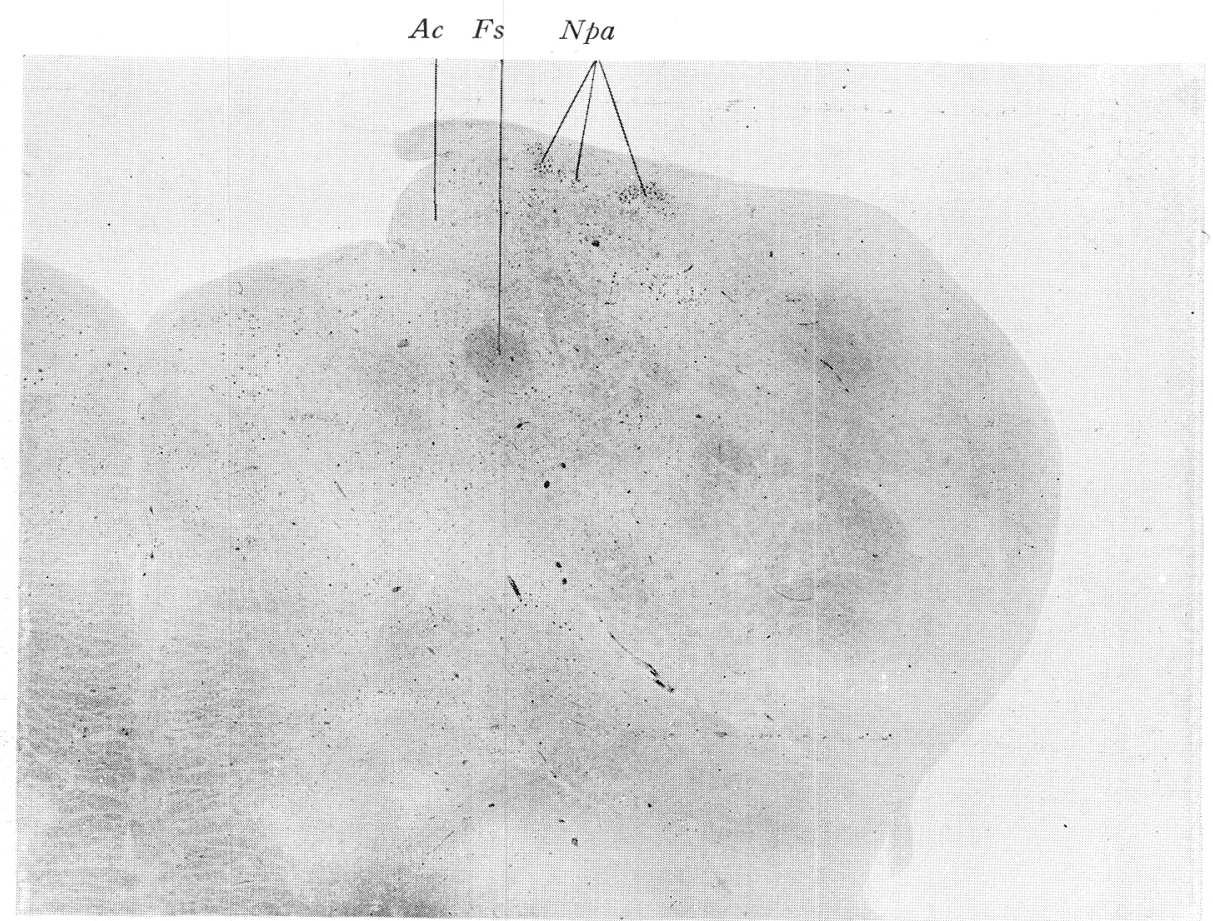

Fig. 2. Transverse section of the medulla, corresponding to fig. 1, from a case with lesions of the $L_{2}-S_{5}$ posterior roots (case of Dogliotti's subarachnoidal alcohol injection). Degeneration was traced exclusively in Goll's funiculus of one side. Note the small mass of fine Marchi-granules ( $N p a$ ) just lateral to the lateral margin of the ala cinerea $(A c)$. $F s$, solitary fascicle. Marchi-stain, $50 \mu$ thick.

That these cells, however, show considerable differences in form and size from those of the proper triangular nucleus has been pointed out by Goldowski (5) and they can be easily distinguished from the cells of the proper gracile nucleus too, for they are far smaller than the latter. Hence, the opinion is tenable that in the close neighbourhood of the ala cinerea between the caudal part of the triangular nucleus and the rostral tip of Goll's nucleus a special grey matter exists, in which a part of the fibers from the sacral cord terminate. 
These investigations have thus elucidated the existence of two different kinds of centripetal connections between the sacral cord and the medulla oblongata in man, one ascending in the lateral funiculus and the other in the posterior funiculus.

In view of the supposed primitive function (mediation form the pelvic cavity of the visceral sensation including the sexual) of these systems we expected the existence of analogous ones among all vertebrates.

Ogiya (16) in our laboratory, prepared a series of hemi-sections and transections of the lower part of the spinal cord $\left(L_{2^{-}} S_{1}\right)$ of the cat (whose 7 th lumbar, 1st, 2nd and 3rd sacral roots correspond to the 2nd to 4 th sacral in

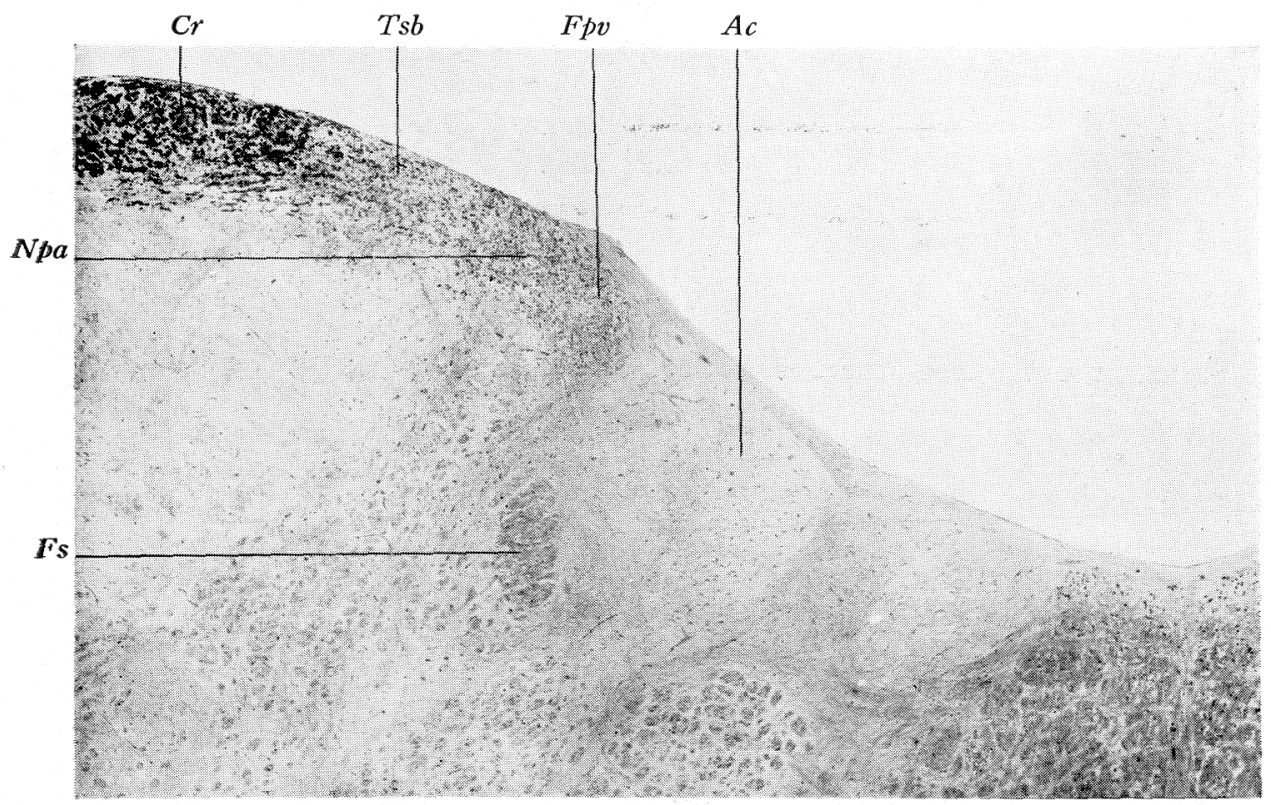

Fig. 3. Transverse section of the middle part of the medulla from a cat, whose cord was hemisectioned at the level of the 2 nd lumbar segment 8 days previously. Note extremely fine degenerated fibers ( $T s b)$ emerging from the restiform body $(\mathrm{Cr})$ and terminating in a grey matter $(N p a)$ in close proximity to the dorsal nucleus of the vagus $(A c)$. The medial half of this grey matter is filled chiefly by fibers from the posterior funiculus $(\boldsymbol{F} p v)$. Compare with fig. 4. Marchi-stain, $50 \mu$ thick.

man), and managed to trace 3 kinds of degenerated fibers ascending to the border of the 4th ventricle, the one, forming a component of the lateral funiculus, may be compared to the sacro-bulbar tracts in man, while the other two, participating in the formation of the posterior funiculus, assume in certain respects the ordinary component of the posterior funiculus, but they penetrate Goll's nucleus in a strict sense and ascend further cephalad. One of the latter terminates in the small grey matter near the most medial margin of the restiform body at the level of the entrance of the glossopharyngeal nerve. 
It is difficult to say whether it should be interpreted as the rostral end of the other, as its relation to the dorsal nucleus of the vagus appears to be not so intimate. The other group of fibers, on the contrary, which certainly originates from the sacral posterior root ganglia, terminates in a grey mass (fig. 3 , which lies in close proximity to the dorsal nucleus of the vagus just beneath the tela choroidea of the 4 th ventricle and whose caudal extension seems to occupy the medio-dorsal margin of Goll's nucleus (therefore, in the caudal part it also keeps the same relation to the caudal extension of the dorsal nucleus

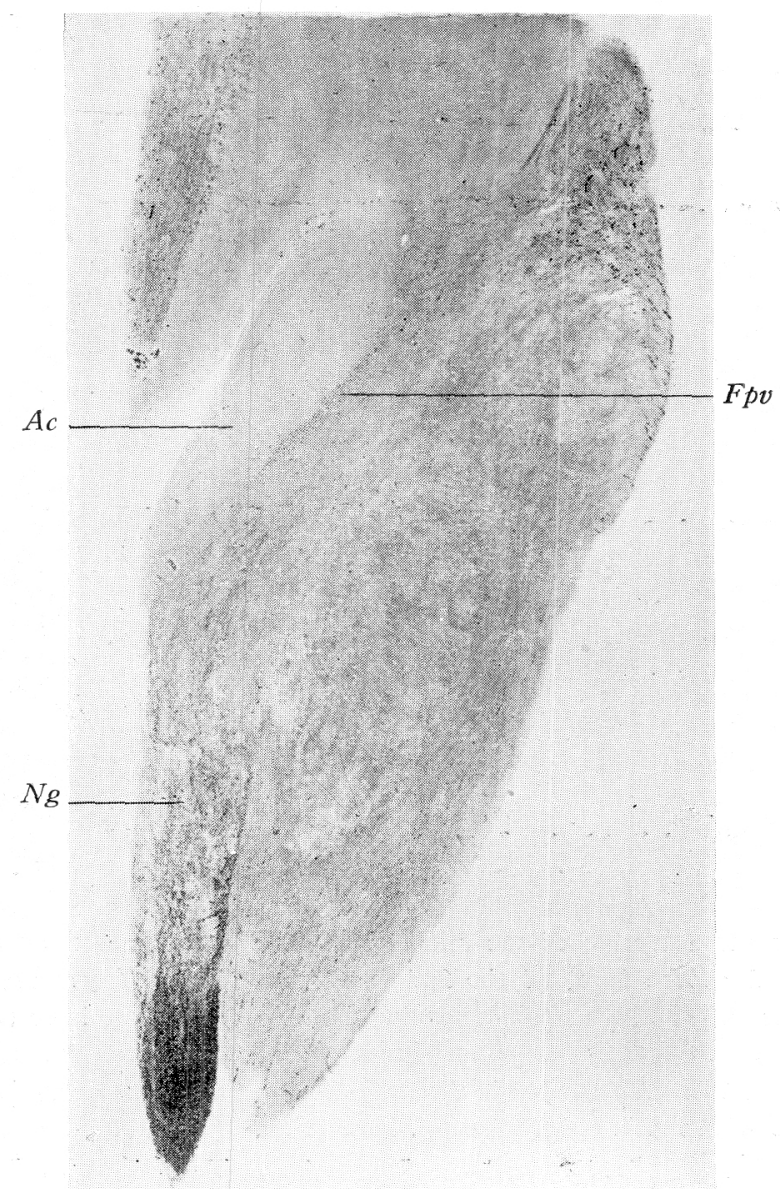

Fig. 4. Longitudinal section of the medulla of a cat, whose cord was cut transversely at the level of the 5 th lumbar segment 13 days previously. Extremely fine degenerated fibers $(F p v)$ pass along the border of the ala cinerea $(A c)$ from the gracile nucleus $(N g)$ which is filled with more coarse granules. Marchi-stain, $50 \mu$ thick.

of the vagus). In Nissl preparations the cells of this grey substance can be easily differentiated by their size and from, both from those of the true triangular nucleus and from those of Goll's nucleus in the ordinary sense. I wish to name this grey matter "nucleus paraalaris." 
That, strictly speaking, this part of grey matter should not be included in the gracile nucleus, can be easily shown also from investigations of Marchipreparations of rhizotomy experiments $\left(L_{7}-S_{3}\right)$ in longitudinal series of section; the rather thick Marchi-granules stop a little rostral to the obex filling Goll's nucleus in the strict sense, while a mass of much finer granules ascends further rostrally, bordering continuously the lateral margin of the ala cinerea and terminates in the grey matter above mentioned (fig. 4). Its relation to the vestibular nerve can also be easily excluded by tracing the degeneration caused by the destruction of the labyrinth.

From these investigations we reached the conclusion, that in man, as well as in cat, a special component of the posterior funiculus exists, which originates in the posterior root ganglia of the inferior cord and terminates by the side of the floor of the 4th ventricle in a certain mass of grey substance (nucleus paraalaris) situated directly lateral to the dorsal nucleus of the vagus.

To obtain further information about these circumstances we chose animals with simple constitution of the dorsal funiculus (Rana catesbiana Shaw, Rana nigromaculata Hollowell, and Bufo vulgaris japonicus Schlegel) and made transections of the inferior part of their spinal cord. In these animals :

1) The adult forms have no tail and consequently the ascending as well as the descending connections concerning the movement of the tail can be excluded.

2) Most of the fibers in the posterior funiculus are said to connect the adjacent spinal segments with each other or to be the descending fibers from the cranial nerves (Wallenberg (18)).

3) The medial lemniscus has not been proved to be present and accordingly the existence of the posterior funiculus nuclei in the usual sense is doubted (Ariens Kappers, Huber and Crosby(1)).

The last point deserves especial note, for if a long ascending system be found in the posterior funiculus of these animals, it should not be interpreted as the antecedent of the bulbo-thalamic fibers in the medial lemniscus but may signify another functional unit.

As in the higher vertebrates, if we call those roots which come out between the cranium and the first vertebra the first pair, this disappears in frogs in the course of metamorphosis and we can distinguish 10 pairs (2nd to 11th) of posterior roots in the adult from, each of them emerging behind the vertebra of the same number. Of these the 8th, 9 th and 10th roots form the plexus lumbosacralis and the last two (10th and 11th) form the ischio-coccygeal plexus. These plexuses correspond to the lumbo-sacral and pudendal in higher vertebrates and fibers from the ischio-coccygeal plexus innervate, besides $M$. compressor cloacae and M. sphincter ani, rectum, bladder, genitals and the coccygeal lymphatic heart (Gaupp (4)).

Konaka (7) in our laboratory made transections of the upper portion of the 10th segment of these animals and traced the ascending degenerations in Marchipreparations. Besides the well known degenerations in the lateral and anterior fascicles a conspicuous cuneiform mass of degenerated fibers is seen in the most medial part of the posterior funiculus just lateral to the septum medianum 

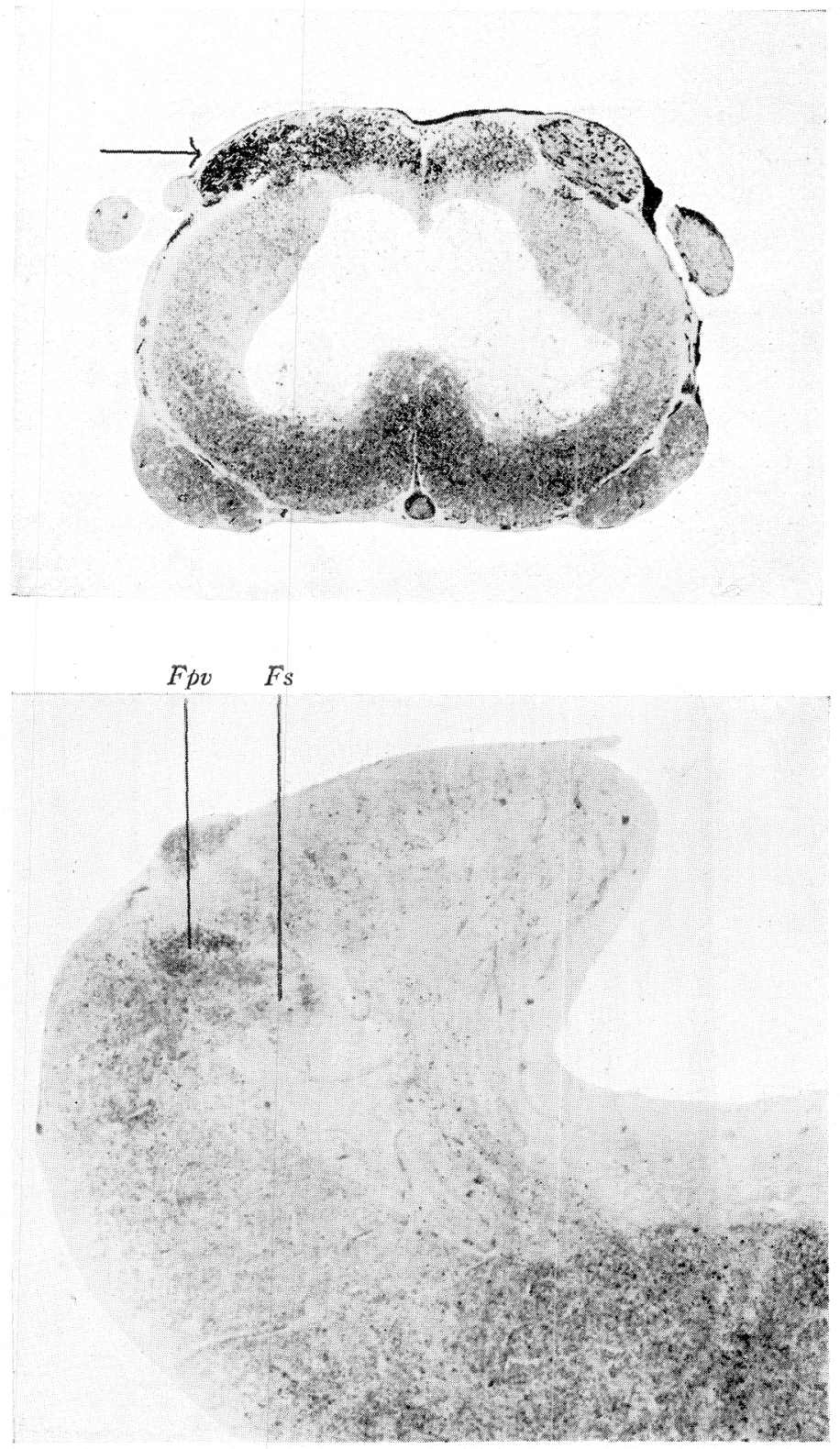

Fig. 5. Upper: Section of the 10th spinal segment of a frog (Rana catesbiana Shaw), whose right 10 th posterior root (marked with arrow) was cut inside the spinal canal 13 days previously. (The slight degeneration of the opposite root may have been caused by the stretching during the operation).

Below: A conspicuous triangular area full of Marchi-granules is seen just lateral to the solitary fascicle $(F p v)$. Section of the medulla from the same frog a little caudal to the entrance of the vagus nerve. Marchi-stain, $50 \mu$ thick. 

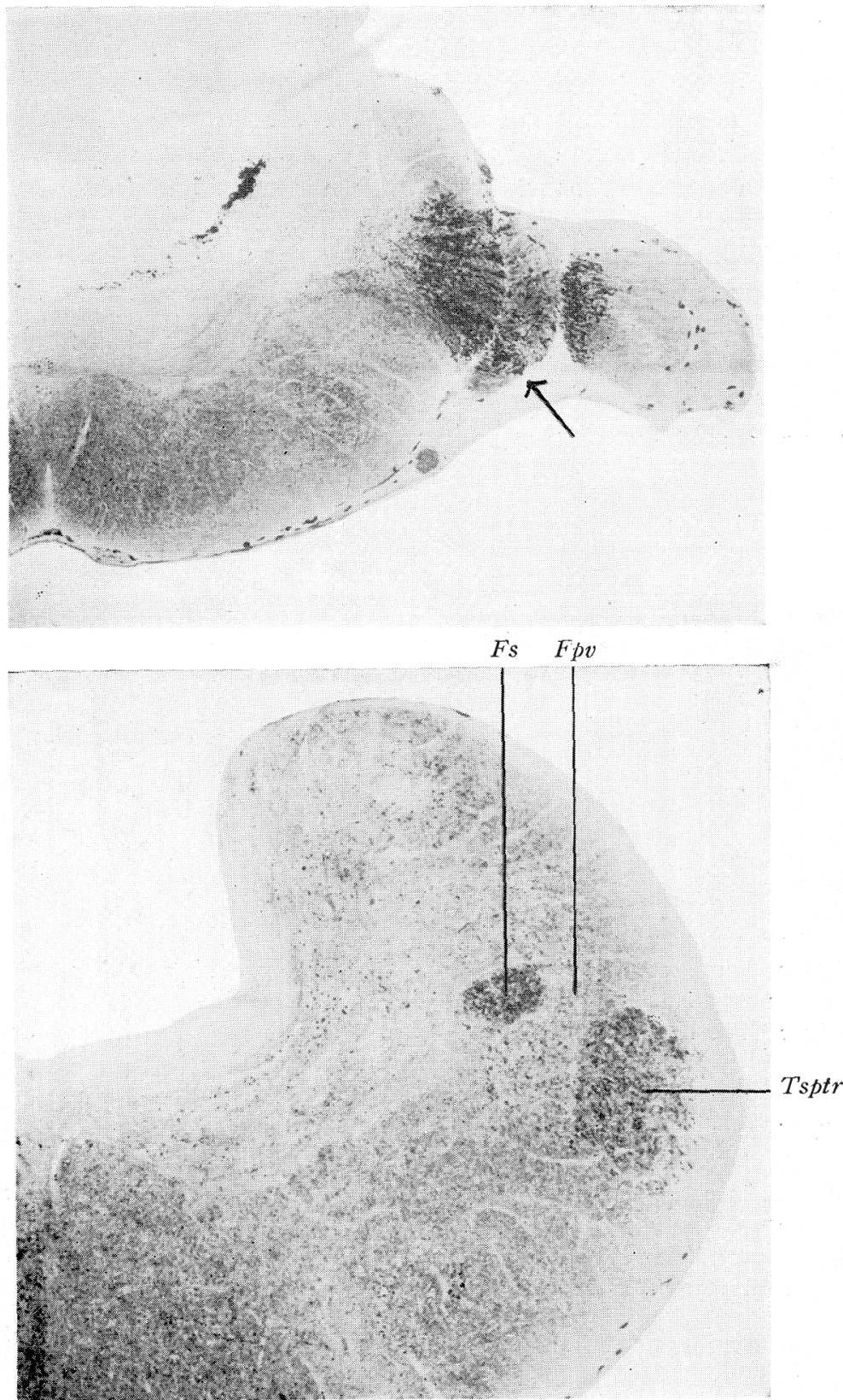

Fig. 6. Upper: Section of the brain stem at the level of the entrance of the facial and trigeminal nerves of a frog (Rana catesbiana Shaw), whose left facial and trigeminal nerves were cut within the cranium (marked with arrow).

Below: Section of the medulla from the same frog a little rostral to the entrance of the vagus nerve. The intact fascicle of the pelvic vagus $(F p v)$ is clearly contrasted with the degenerated area of the solitary fascicle $(F \mathrm{~s})$, which is composed of the facial root fibers at this level, and the spinal tract of trigeminal nerve (Tsptr). Marchi-stain, $50 \mu$ thick, 
posterius. In the 2nd cervical segment (where the most caudal portion of the solitary fascicle is seen already) it is situated just dorsal to the solitary fascicle and always keeping a dorsal or dorso-lateral position to the solitary fascicle, shifts gradually lateral with the deviation of the latter itself in the inferior half of the rhombencephalon. At the level of the entrance of the vagus nerve (which forms a common root with the glossopharyngeal and accessory nerves) it lies so closely to the solitary fascicle (fig. 5) that in Weigert-preparations it can scarcely be disitinguished from the latter. Further rostral it disappears rapidly with the diminution of the solitary fascicle itself and above the level of the entrance of the facial nerve it is no longer traceable. This type of degeneration is observed almost to the same degree in cases of transections of higher segments and also after a single section of the 10th posterior root within the spinal canal (fig. 5). Thus the conclusion is tenable that most of the conspicuous ascending
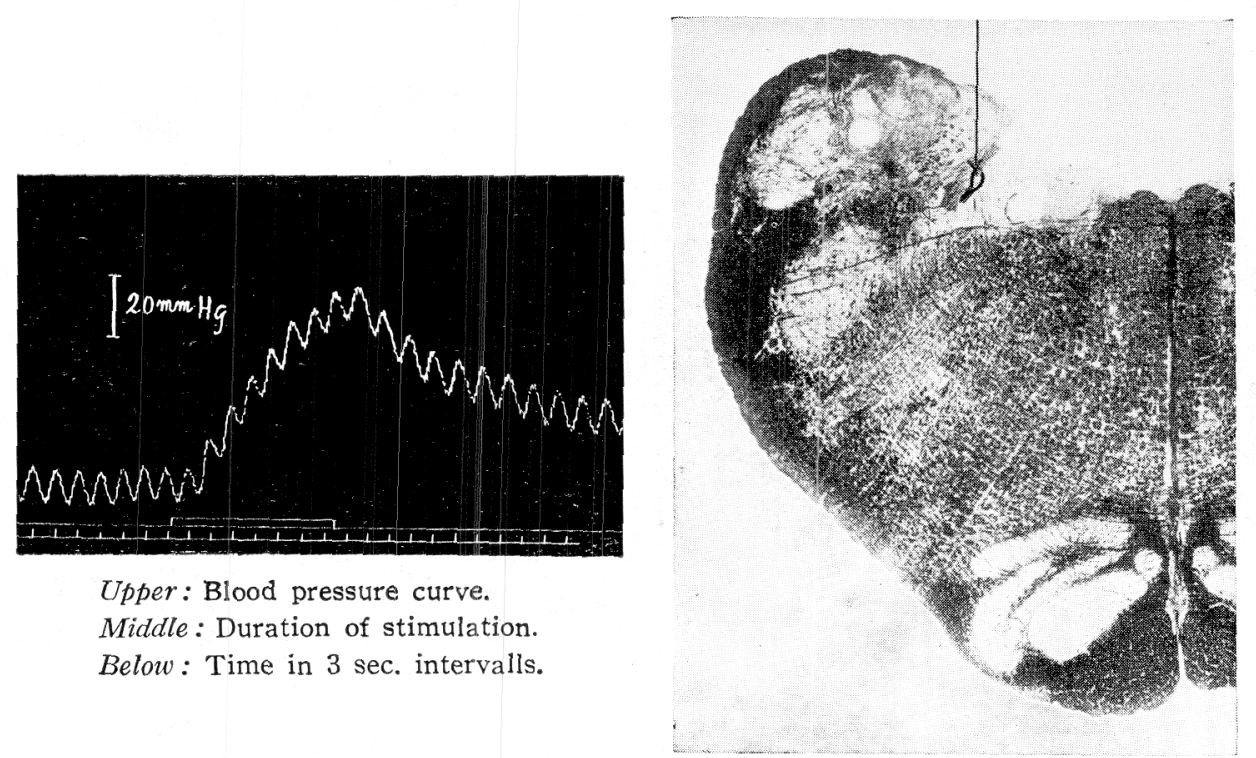

Upper: Blood pressure curve. Middle: Duration of stimulation. Below: Time in $3 \mathrm{sec}$. intervalls.

Fig. 7. A conspicuous rise in blood pressure can be constantly recorded by stimulating a part closely adjacent to the solitary fascicle at the level of the upper-most part of the ala cinerea in cat. Thickness of the electrode $0.08 \mathrm{~mm}$. The stimulating current was supplied from a $0.1 \mu \mathrm{fd}$. condenser discharge regulated with thyratron. Intensity about 0.3 volt and frequency about 30/sec. After the recording the spot is marked with a $0.1 \mathrm{~mA}$ direct current discharge for $4 \mathrm{sec}$. (Right half : arrow).

fibers in the medial part of the posterior funiculus have their origin in the 10th posterior root, in these animals. In other words, in frogs the nerve cells, whose axons ascend in the middle-most part of the posterior funiculus and finally fuse completely with the solitary fascicle in the medulla, exist mainly in the 10th posterior root ganglion and, therefore, their peripheral branches innervate the rectum, bladder, genitals and cloaca.

As above mentioned, in the posterior funiculus of these animals are contained a series of descending fibers. Wallenberg (18) could clearly demonstrate that in 
lower vertebrates, the trigeminal, vestibular and vagus nerves send axons deep into the spinal cord. To exclude the eventual appearance of retrograde degeneration, Tatumi (17) in our laboratory performed series of experiments in which he cut the vagus, trigeminal, facial and acoustic nerves separately or in combinations. The results showed clearly that all of the descending fibers from these nerves occupy more lateral situations in the cord (as already described by Wallenberg (18) ) and exclude their confusion with the medial ascending fibers from the 10th posterior root. One of these preparations, in which the site in the medulla of the ascending component of the inferior cord is clearly demonstrated between degenerated fibers of the solitary fascicle (which at this level is mainly composed of the descending fibers of the facial nerve) and those of the spinal tract of the trigeminal nerve, is presented in fig. 6 . Compare wlth fig. 5 and note the contrast just in mirror image.

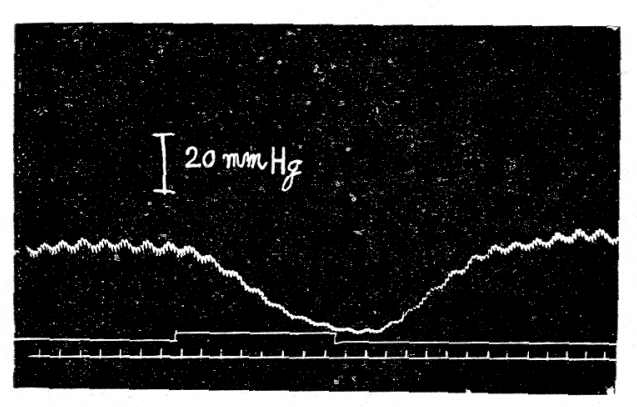

Upper: Blood pressure curve.

Middle: Duration of stimulation.

Below: Time in $3 \mathrm{sec}$, intervalls.

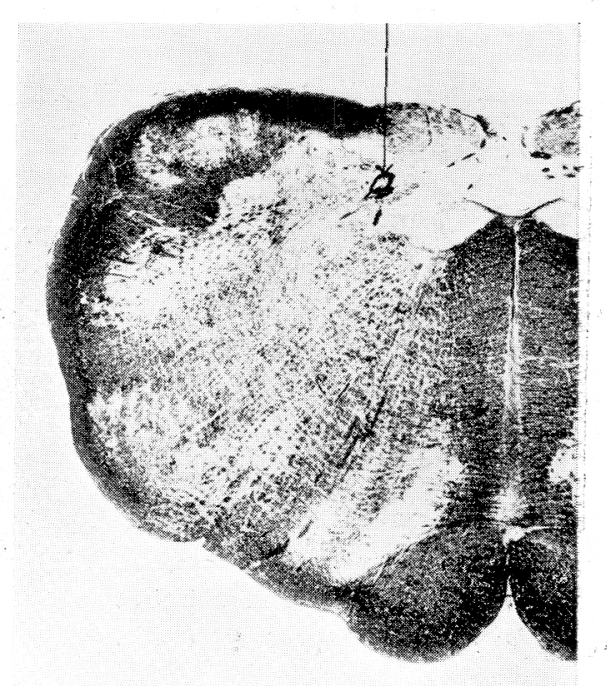

Fig. 8. A remarkable fall in the blood pressure can be usually obtained in cat by stimulating a part closely adjacent to the solitary fascicle at the level of the obex. As to the conditions of stimulation, consult the note in fig. 7. On the right half the stimulated spot is marked with an arrow.

This type of ascending fibers in the middle-most part of the posterior funiculus in frogs, can be regarded as the pelvic equivalent of the sensory portion of the vagus nerve (or the pelvic vagus for short), for:

1) their peripheral branches innervate the viscera in the pelvic cavity (rectum, bladder, genitals and cloaca), as the peripheral branches of the vagus nerve do those in the thoracoabdominal cavity,

2) the rostral part of the central branches fuse in the solitary fascicle, and

3) they belong to the protoneurons, similar to axons of the sensory vagus, and consequently do not cross throughout their whole course in the spinal cord and the medulla. 
Keeping in consideration these simple conditions in frogs, and review once more those in man and cat, the analogy can be at once fathomed. The ascending fibers in the middle-most part of the posterior funiculus in these higher vertebrates are also connected to the pelvic organs via sacral or pudendal plexus, ascend undecussated in the posterior funiculus, terminate in the nucleus closely adjacent to the dorsal or sensory (solitary fascicle) nucleus of the vagus nerve and do not ascend beyond the level of the entrance of the glossopharyngeal nerve. The slight difference exists in the fact, that in man as well as in cat these fibers are not confluent with the solitary fascicle itself. Nevertheless, that they signify an analogous connection, can be concluded from the fact that the other crossed connections between the sacral and bulbar autonomic centers through the lateral fascicle (sacro-bulbar tracts) find also their terminations in the same part of the medulla, actually at a certain level co-occupying one and the same

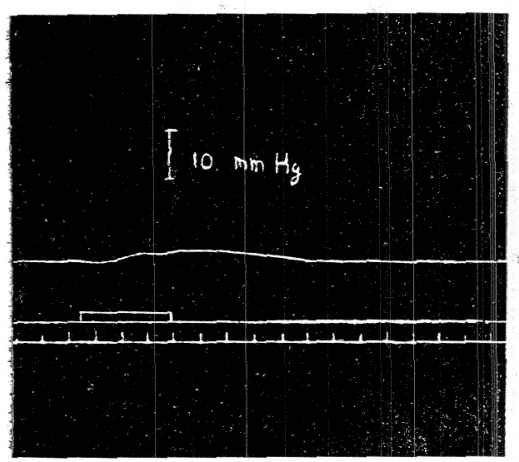

Upper: Blood pressure curve.

Middle: Duration of stimulation.

Below: Time in $3 \mathrm{sec}$. intervalls.

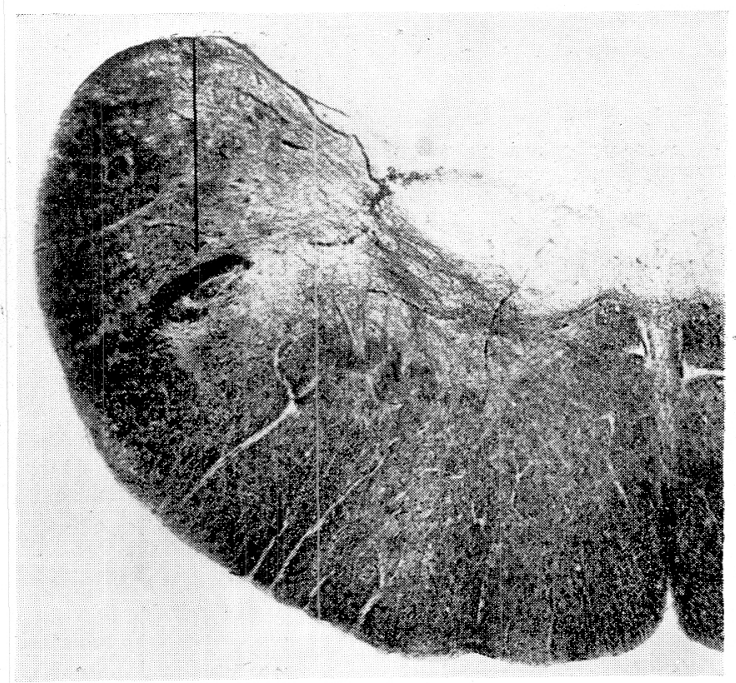

Fig. 9. Pressor point in bull-frog. As to conditiors of stimulation consult the note in fig. 7. The stimulated point is illustrated in the right half by an arrow. The direction of the electrode can be traced as a stab canal, whose end just indicates the site of the pelvic vagus.

grey substance.

Langley $(11,12)$ succeeded in pointing out the existence of 2 kinds of centrifugal fibers (the sympathetic and parasympathetic) to viscera, which stand in antagonistic relation, and Ishikawa (6) and his collaborators endeavoured to expand this concept into the sensory half of the visceral innervation. As further investigations have shown, however, the sympathetic and parasympathetic systems do not present always a diagonal function, they inter- and coact in many instances (Langworthy (13)). Meanwhile, the bulbar and sacral autonomic systems are characterized by having their own vasodilatatory centers, and consequently the excitation of one should act inhibitorily on the other, and, moreover, the marked local congestion should be due to the general elevation of blood pres- 
sure. Thus the existence of ascending connections from the sacral autonomic center to the bulbar is as highly probable as their termination in the close neighbourhood of the bulbar vasomotor centers.

In previous communications $\mathrm{I}(8,9,10)$ pointed out one of these systems in man (sacro-bulbar tracts), which are characterized by their origin in the sacral segments, by their crossed course in the spinal cord and by their termination in the close vicinity of the solitary tract, and later experiments of Ogiya, as above mentioned, have shown the existence of an analogous system in cat. Here I have described a second connection which ascends in the posterior funiculus (pelvic vagus).

In utilizing a fairly fine unipolar electrode (diameter $80 \mu$ ) and supplying the stimulus from a $0.1 \mu \mathrm{fd}$. condenser discharge regulated by a thyratron, Yamamoto (21) in our laboratory reinvestigated Wann and Ranson's (19) experiment. According to him the distribution of the bulbar vasomotor centers is, unlike the conclusions reached by the latter, in a well localized part corresponding to the

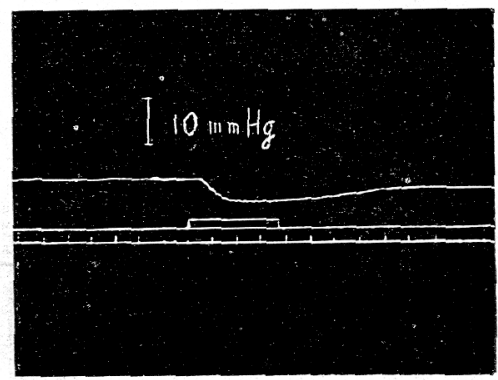

Upper: Blood pressure curve.

Middle: Duration of stimulation.

Below: Time in 3 sec. intervalls.

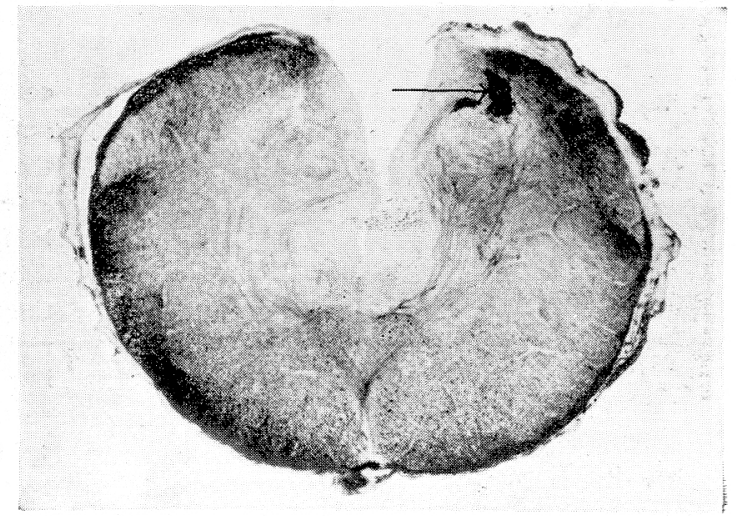

Fig. 10. Depressor point in bull-frog. As to conditions of stimulation consult the note in fig. 7. The stimulated point is indicated in the right half by an arrow.

course of the solitary fascicle or along the border of the ala cinerea, that is to say, in the close vicinity of the terminations of the sacro-bulbar connections (fig. 7 and 8). Employing the same fine electrode, I with Tatumi endeavoured to locate the site of the vasomotor centers in the bull-frog; analogous to conditions in the cat, these centers are localized near the neighbourhood of the solitary fascicle, i.e., near the termination of the pelvic vagus (fig. 9, 10 and 11). This result seems to me to be significant to our hypothesis above, that between the bulbar and sacral parasympathetic centers, in other words, between both autonomic centers with their own vasodilators, should exist centripetal connections and in the neighbourhood of their terminations the bulbar vasomotor centers exist. To the pelvic equivalent of the sensory vagus should be attributed a function analogous to that of the sensory vagus, and therefore, it may probably be connected to the bulbar parasympathetic center. And if this be the case, the connection of the sacro-bulbar systems to the medullary sympathetic center can also be alluded to. 
Conclusion. A special component of the posterior funiculus has been demonstrated, which deserves the name "the pelvic equivalent of the sensory vagus" or for short "the pelvic vagus." The nerve cells of this system exist probably in the 2nd, 3rd and 4th sacral posterior root ganglia in man, in the 7th lumbar and 1st to 3rd sacral posterior root ganglia in cat and surely in the last two (10th and 11th) posterior root ganglia in frogs. Their peripheral branches innervate the visceral organs in the pelvic cavity (rectum, bladder, gerritals and in frogs also the cloaca), while the central ones ascend in the middle-most portion of the posterior funiculus and terminate in the medulla in the grey substance lateral to the dorsal nucleus of the vagus in man and cat and perhaps in the common grey substance accompanying the solitary fascicle in frogs.

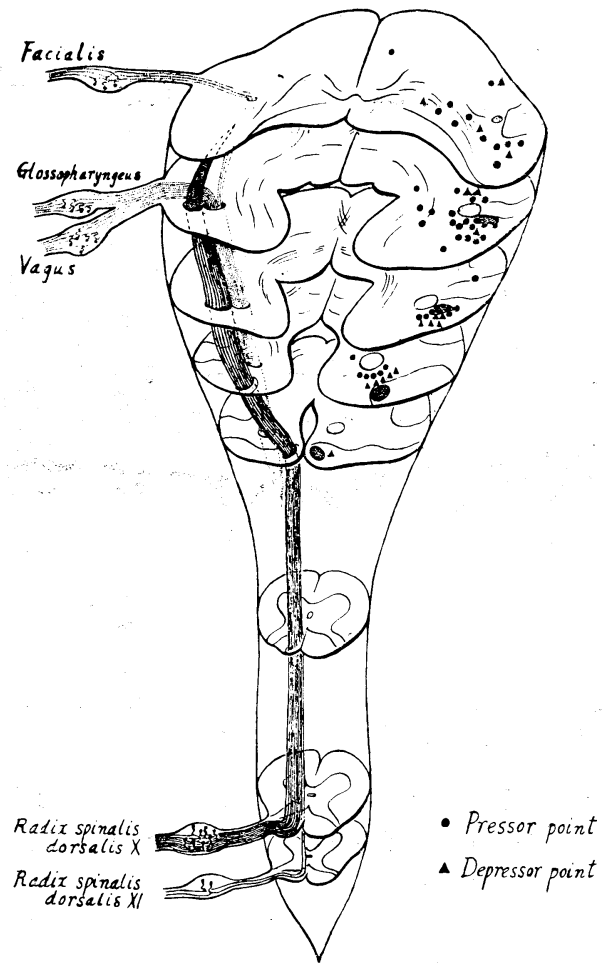

Fig. 11. Diagram showing the course of the solitary fascicle (dotted) and the pelvic vagus (in full lines) [in the left half] and their relation to the bulbar vasomotor centers [in the right half] in bull-frog. The reaction obtained in 30 animals on both side of the medulla was transferred to the right half of the diagram superimposing the vasomotor points in about 15 sections on one plate. 15 isolated points in the ventral half of the preparations are not visible in this diagram.

The other connection between the inferior part of the cord and medulla (sacro-bulbar tracts), which was previously described by me, terminate partially in the same nucleus and its vicinity, and in the neighbourhood of the terminations of these two connections, the existence of the bulbar vasomotor centers can be confirmed. An antagonistic (functionally also synergic or corelative) relation analogous to that between the sympathetic and parasympathetic can be assumed to exist between these two centripetal connections.

The expenses for this work were defrayed by a grant for the scientific researches from the Department of Education. 


\section{REFERENCES}

1. ARIENS KAPpers, C. U., G. C. Huber AND E. C. CROSBY. The comparative anatomy of the nervous system of vertebrates, including man. New York 1936.

2. Foerster, O. AND O. GAGel. Z. Neur. 138: 1-90, 1932.

3. Fröhlich, A. AND H. H. MEYer. Z. exper. Med. $29: 87-113,1922$.

4. GAUPP, E. Anatomie des Frosches I-III. Braunschweig 1896-1904.

5. GoldowsKI, W. J. Arb. neur. Inst. Wien. 32: 289-340, 1930.

6. IshIKAW A; H. Quoted from S. ARAKI, Rinsyo no simpo. Vol. 1. Osaka 1949.

7. KonAKA, T. To appear in Zyuzenkaizassi.

8. KURU, M. Jap. J. Med. Sci. Part I, Anat. 8: 135-160, 1940.

9. - Sensory paths in the spinal cord and brain stem of man. Tokyo 1949.

10. KURU, M. AND B. TAKASE. Fol. psychiatr. neur. Jap. 2 : 124-151, 1947.

11. LANGley, J. N. Brain $26: 1-26,1903$.

12. - Autonomic nervous system. Cambridge 1921.

13. Langworthy, O. R. Arch. Neur. 50:590-602, 1943.

14. MARBURG, O. Mikroskopisch-topographischer Atlas des menschlichen Zentralnervenstems. 3. Aufl. Leipzig and Vienna 1927.

15. NAKAMURA, T. Zyuzenkaizassi $52: 430-445,1950$.

16. OGIYA, R. To appear in Zyuzenkaizassi.

17. TATUMI, K. To appear in Zyuzenkaizassi.

18. Wallenberg, A. Anat. Anz. 30:564-568, 1907.

19. WANN, S. C. AND S. W. RANSON. J. comp. Neur. $71: 437-455,1939$.

20. White. J. C. Res. Publ. Ass. nerv. ment. Dis. 23 : 373-390, 1943.

21. YАмАмото, S. To appear in Zyuzenkaizassi.

22. ZIEHEN, Th. Bardeleben's Handbuch der Anatomie des Menschen. Nervensystem II Abt. Jena 1934. 